A simple example illustrating the application of thermodynamic extremum principles

This article has been downloaded from IOPscience. Please scroll down to see the full text article.

2002 Eur. J. Phys. 23501

(http://iopscience.iop.org/0143-0807/23/5/307)

View the table of contents for this issue, or go to the journal homepage for more

Download details:

IP Address: 147.96.14.15

The article was downloaded on 06/06/2013 at 12:01

Please note that terms and conditions apply. 


\title{
A simple example illustrating the application of thermodynamic extremum principles
}

\author{
S Velasco ${ }^{1}$ and C Fernandez-Pineda ${ }^{2}$ \\ ${ }^{1}$ Departamento de Física Aplicada, Facultad de Ciencias, Universidad de Salamanca, \\ 37008 Salamanca, Spain \\ 2 Departamento de Física Aplicada I, Facultad de Físicas, Universidad Complutense, \\ 28040 Madrid, Spain
}

Received 5 March 2002, in final form 21 May 2002

Published 8 August 2002

Online at stacks.iop.org/EJP/23/501

\begin{abstract}
A composite system consisting of two identical ideal gases separated by a movable, frictionless, diathermic piston is used to illustrate the application of different extremum principles in thermodynamics. The time evolution of the system from an initial constrained equilibrium state to the final equilibrium state is analysed by means of a very simple phenomenological model.
\end{abstract}

\section{Introduction}

There are several extremum principles in thermodynamics. When teaching thermodynamics to undergraduates, attention is frequently restricted to the following ones.

(I) The entropy maximum principle. The equilibrium value of any unconstrained internal variable is such as to maximize the entropy $(S)$ for given values of the total internal energy $(U)$, volume $(V)$ and mole number $(n)$.

(II) The internal energy minimum principle. The equilibrium value of any unconstrained internal variable is such as to minimize the internal energy for given values of the total entropy, volume and mole number.

These principles refer to hydrostatic (or $P V T$ ) closed systems and they were formulated by Gibbs, who also proved the equivalence between them by showing that a violation of one leads to a violation of the other [1]. A discussion of the principles, including different proofs of their equivalence, can be found in most textbooks of thermodynamics [2-5].

A less known third extremum principle, not considered by Gibbs, can be formulated for $P V T$ systems.

(III) The volume minimum principle. The equilibrium value of any unconstrained internal variable is such as to minimize the volume for given values of the total entropy, internal energy and mole number.

The validity of this principle has been established by Kazes and Cutler [6] (see, also, [7, 8]). 
The mathematical formulation of the above thermodynamic extremum principles parallels the formulation of similar principles in mechanics. In the equilibrium state one verifies either $(\delta S)_{U, V, n} \leqslant 0$, or $(\delta U)_{S, V, n} \geqslant 0$, or $(\delta V)_{S, U, n} \geqslant 0$, where the symbol $\delta$ denotes a virtual (or variational) change in the same sense as the virtual displacements in mechanics. The extremum principles are then applied to obtain the equilibrium conditions of a given composite system, initially in a constrained equilibrium state, and to yield the sign of certain thermodynamic coefficients (stability conditions). This procedure is very general since it does not require knowledge of explicit expressions of the fundamental equations of the involved systems. However, the practical application of the extremum principles for the determination of the equilibrium state of concrete systems can also be of didactic value, e.g. for analysing its extremum character (maximum or minimum) or for comparing the results obtained from the application of the different principles.

Very recently [9] we have elucidated the three above, somewhat abstract, extremum principles for a simple physical system proposed by Bazarov [10]. This system consists of an ideal gas interacting with a constant-pressure work device through an movable adiabatic piston. From a mathematical point of view this model has the pedagogical value of its simplicity since the system considered needs only one variable for describing its equilibrium state. Unfortunately, it only allows us to discuss the mechanical equilibrium condition of equal pressures. The aim of the present work is to apply the extremum principles to a more general system exhibiting both thermal and mechanical interactions. In section 2 the system is described from a thermodynamic viewpoint. In section 3 we apply the extremum principles for the entropy (I), the internal energy (II) and the volume (III) in order to identify the different equilibrium states. This allows us to obtain the final equilibrium state and to analyse its extremum character. Numerical results are reported for a particular case, illustrating the behaviour of the system along different variational processes. In section 4 we present a simple phenomenological model for the considered system. This model predicts the same final equilibrium state as reported in section 3, and allows one to analyse qualitatively the time evolution of the system from an initial constrained equilibrium state toward the final equilibrium state.

\section{The model}

Consider the system illustrated in figure 1. An adiabatic cylinder contains an internal piston which divides the volume into two chambers, labelled 1 and 2, containing $n_{1}$ and $n_{2}$ moles, respectively, of the same ideal gas. Denoting the volumes of the left and right chambers by $V_{1}$ and $V_{2}$, respectively, and the corresponding temperatures by $T_{1}$ and $T_{2}$, the pressure, internal energy and entropy of the gases are assumed to be [11]

$$
\begin{aligned}
& P_{1}=\frac{n_{1} R T_{1}}{V_{1}}, \quad P_{2}=\frac{n_{2} R T_{2}}{V_{2}}, \\
& U_{1}=c n_{1} R T_{1}, \quad U_{2}=c n_{2} R T_{2}, \\
& S_{1}=S_{0,1}+c n_{1} R \ln T_{1}+n_{1} R \ln V_{1}, \quad S_{2}=S_{0,2}+c n_{2} R \ln T_{2}+n_{2} R \ln V_{2},
\end{aligned}
$$

where $R$ is the molar gas constant, $c$ is a positive constant related to the molar heat capacity at constant volume of the gas $\left(c=c_{\mathrm{V}} / R\right)$ and $S_{0,1}$ and $S_{0,2}$ are constants.

Initially, the piston is adiabatic and rigidly fixed by latches and the two subsystems are in equilibrium states characterized by $\left(T_{1, i}, V_{1, i}\right)$ and $\left(T_{2, i}, V_{2, i}\right)$, with $T_{1, i} \neq T_{2, i}$ and $V_{1, i} \neq V_{2, i}$. At a certain time, the latches are released and the adiabatic constraint is removed so that the piston becomes a movable, frictionless, diathermic piston. As is well known, thermodynamics predicts that the system evolves toward a unique final equilibrium state, independent of the sequence in which the constraints are removed, characterized by the equality of the temperatures and pressures on both sides. Our aim is to analyse this final equilibrium state from the viewpoint of the extremum principles for the total entropy, internal energy and volume. 

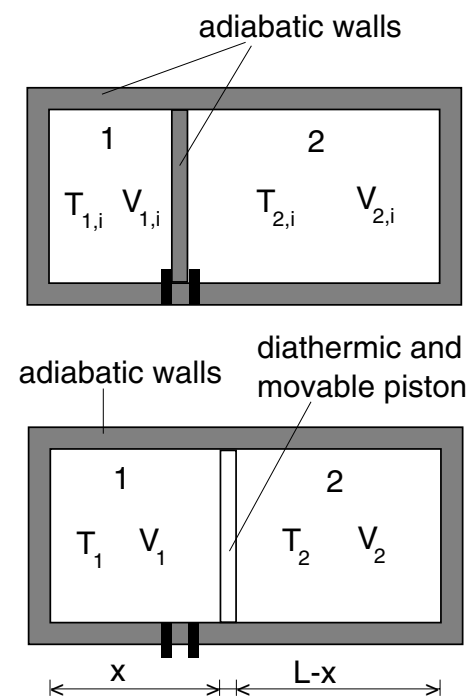

Figure 1. The system under study.

\section{Application of the extremum principles}

\subsection{Entropy maximum principle}

From equations (3), the entropy of the total system is given by

$$
S=S_{1}+S_{2}=S_{0}+c R\left(n_{1} \ln T_{1}+n_{2} \ln T_{2}\right)+R\left(n_{1} \ln V_{1}+n_{2} \ln V_{2}\right),
$$

where $S_{0}=S_{0,1}+S_{0,2}$ is a constant. Furthermore, since the internal energy and the volume of the total system remain constant one has

$$
\begin{aligned}
& U_{1}+U_{2}=c R\left(n_{1} T_{1}+n_{2} T_{2}\right)=\text { constant } \\
& V_{1}+V_{2}=\text { constant. }
\end{aligned}
$$

Equations (5) and (6) show that the equilibrium states of the considered composite system can be described by means of two internal variables, i.e. there are two independent variables in the maximization process for the entropy (4) under external constraints (5) and (6). Let us take the temperature $T_{1}$ and the volume $V_{1}$ as these variables. From equations (5) and (6) one has

$$
\begin{aligned}
& T_{2}=\frac{A-n_{1} T_{1}}{n_{2}}, \\
& V_{2}=B-V_{1},
\end{aligned}
$$

respectively, where $A$ and $B$ are constants that can be calculated from the initial temperatures and volumes,

$$
\begin{aligned}
& A=n_{1} T_{1, i}+n_{2} T_{2, i}>0, \\
& B=V_{1, i}+V_{2, i}>0 .
\end{aligned}
$$

Substituting equations (7) and (8) into (4) yields

$\left(S-S_{0}\right)_{U, V} \equiv S\left(T_{1}, V_{1}\right)=c R\left[n_{1} \ln T_{1}+n_{2} \ln \left(\frac{A-n_{1} T_{1}}{n_{2}}\right)\right]+R\left[n_{1} \ln V_{1}+n_{2} \ln \left(B-V_{1}\right)\right]$. 
The condition $\left(\delta^{1} S\right)_{U, V}=0$ for which the entropy attains a local extremum at $\left(T_{1, e}, V_{1, e}\right)$ requires that the two first partial derivatives of the function (11) vanish. This requirement allows one to obtain

$$
\begin{aligned}
& T_{1, e}=\frac{A}{n}=\frac{n_{1} T_{1, i}+n_{2} T_{2, i}}{n}, \\
& V_{1, e}=\frac{n_{1} B}{n}=\frac{n_{1}\left(V_{1, i}+V_{2, i}\right)}{n},
\end{aligned}
$$

with $n=n_{1}+n_{2}$, for the temperature and the volume of gas 1 in the final equilibrium state in terms of the temperatures and volumes of both gases in the initial state. Substituting equations (12) and (13) into (7) and (8), respectively, yields

$$
\begin{aligned}
& T_{2, e}=A-T_{1, e}=\frac{A}{n}, \\
& V_{2, e}=B-V_{1, e}=\frac{n_{2} B}{n},
\end{aligned}
$$

for the temperature and the volume of gas 2. Comparison between equations (12) and (14) leads to

$$
T_{1, e}=T_{2, e},
$$

i.e. to thermal equilibrium. On the other hand, substitution of equations (12)-(15) into (1) leads to

$$
P_{1, e}=P_{2, e}=\frac{R A}{B}=\frac{R\left(n_{1} T_{1, i}+n_{2} T_{2, i}\right)}{V_{1, i}+V_{2, i}},
$$

i.e. to mechanical equilibrium.

The stability condition $\left(\delta^{2} S\right)_{U, V}<0$ is needed to guarantee the entropy maximum for equilibrium. From the calculation of the second partial derivatives of the function (11) at the equilibrium state $\left(T_{1, e}, V_{1, e}\right)$, one obtains

$$
\left(\delta^{2} S\right)_{U, V}=-\frac{n^{3} R}{n_{2}}\left[\frac{c n_{1}}{A^{2}}\left(\delta T_{1}\right)^{2}+\frac{1}{n_{1} B^{2}}\left(\delta V_{1}\right)^{2}\right]<0,
$$

which shows the maximum character of the final equilibrium state.

\subsection{Internal energy minimum principle}

In order to apply the extremum principle for the internal energy it is necessary to know at least one state of the set of equilibrium states where the minimization process is done. This is the final equilibrium state obtained from the entropy maximum principle. Then we can proceed in the following way. From equations (2), the internal energy of the total system is given by

$$
U=U_{1}+U_{2}=c R\left(n_{1} T_{1}+n_{2} T_{2}\right)
$$

Now the external constraints fix the total entropy and volume. The closure relation for the volume is given by equation (2), while the conservation law for the entropy follows directly from equation (4),

$$
S_{0}+R \ln \left(T_{1}^{c n_{1}} T_{2}^{c n_{2}} V_{1}^{n_{1}} V_{2}^{n_{2}}\right)=\text { constant. }
$$

Therefore, the minimization process for the internal energy (19) under external constraints (2) and (20) can be described by means of two independent variables. As in the preceding case, let us take the temperature $T_{1}$ and the volume $V_{1}$ as these variables. From equations (2) and (20) one has

$$
\begin{aligned}
& V_{2}=C-V_{1}, \\
& T_{2}=\frac{D^{1 / c}}{T_{1}^{n_{1} / n_{2}} V_{1}^{n_{1} / c n_{2}} V_{2}^{1 / c}},
\end{aligned}
$$


where $C$ and $D$ are constants that can be calculated from the equilibrium values $\left(T_{1, e}, V_{1, e} ; T_{2, e}, V_{2, e}\right)$, given by equations (12)-(15),

$$
\begin{aligned}
& C=V_{1, e}+V_{2, e}=B, \\
& D=T_{1, e}^{c n_{1} / n_{2}} T_{2, e}^{c} V_{1, e}^{n_{1} / n_{2}} V_{2, e}=\frac{n_{1}^{n_{1} / n_{2}} n_{2} A^{c n / n_{2}} B^{n / n_{2}}}{n^{(c+1) n / n_{2}}},
\end{aligned}
$$

where $A$ and $B$ are given by equations (9) and (10), respectively. Substituting equations (21) and (22) into (19) yields

$$
U_{S, V} \equiv U\left(T_{1}, V_{1}\right)=c R\left[n_{1} T_{1}+\frac{n_{2} D^{1 / c}}{T_{1}^{n_{1} / n_{2}} V_{1}^{n_{1} / c n_{2}}\left(C-V_{1}\right)^{1 / c}}\right] .
$$

From this function, the requirement of an internal energy minimum for the equilibrium state, so that $\left(\delta^{1} U\right)_{S, V}=0$ and $\left(\delta^{2} U\right)_{S, V}>0$, leads to equations (12) and (13), i.e. to the same equilibrium state $\left(T_{1, e}, V_{1, e}\right)$ as obtained from the entropy maximum principle.

\subsection{Volume minimum principle}

As in the preceding case, the application of the extremum principle for the volume requires the knowledge of the final equilibrium state obtained from the entropy maximum principle or the internal energy minimum principle. The function to be minimized is the total volume

$$
V=V_{1}+V_{2}
$$

while the external constraints fix the total entropy and the internal energy. The constraint for the entropy yields equation (22), while the constraint (5) for the internal energy leads to

$$
T_{2}=\frac{E-n_{1} T_{1}}{n_{2}}
$$

where $E$ is a constant that can be calculated from the equilibrium values $T_{1, e}$ and $T_{2, e}$ given, respectively, by equations (12) and (14). We obtain

$$
E=n_{1} T_{1, e}+n_{2} T_{2, e}=A .
$$

From equations (22) and (27) the volume $V_{2}$ can be expressed as

$$
V_{2}=\frac{n_{2}^{c} D}{T_{1}^{c n_{1} / n_{2}}\left(E-n_{1} T_{1}\right)^{c} V_{1}^{n_{1} / n_{2}}}
$$

with $D$ given by equation (24). Substituting equation (29) into (26) yields

$$
V_{S, U} \equiv V\left(T_{1}, V_{1}\right)=V_{1}+\frac{n_{2}^{c} D}{T_{1}^{c n_{1} / n_{2}}\left(E-T_{1}\right)^{c} V_{1}^{n_{1} / n_{2}}} .
$$

Considering the same procedure as in the preceding extremum principles one obtains that the equilibrium state $\left(T_{1, e}, V_{1, e}\right)$, given by equations (12) and (13), corresponds to a local minimum of the volume function (30).

\subsection{A numerical example}

We consider a composite system consisting of $n_{1}=n_{2}=0.1 \mathrm{~mol}$ of the same monatomic ideal gas $(c=3 / 2)$ with $T_{1, i}=100 \mathrm{~K}, V_{1, i}=0.001 \mathrm{~m}^{3}, T_{2, i}=500 \mathrm{~K}$ and $V_{2, i}=0.002 \mathrm{~m}^{3}$. In this case, equations (9) and (10) yield $A=60 \mathrm{~mol} \mathrm{~K}$ and $B=0.003 \mathrm{~m}^{3}$. Figure 2(a) shows a contour plot of the entropy function (11) for the system considered. One can see that this function has a local maximum at $T_{1, e}=300 \mathrm{~K}$ and $V_{1, e}=0.0015 \mathrm{~m}^{3}$ and these values coincide with the results of equations (12) and (13), respectively, predicted by the entropy maximum principle. Figures 2(b) and (c) show a contour plot of the internal energy function (25) and the volume function (30), respectively. In these functions $C=B, E=A$ and $D=70.65 \mathrm{~K}^{3} \mathrm{~m}^{6}$. Both functions show a local minimum at $T_{1, e}=300 \mathrm{~K}$ and $V_{1, e}=0.0015 \mathrm{~m}^{3}$. 

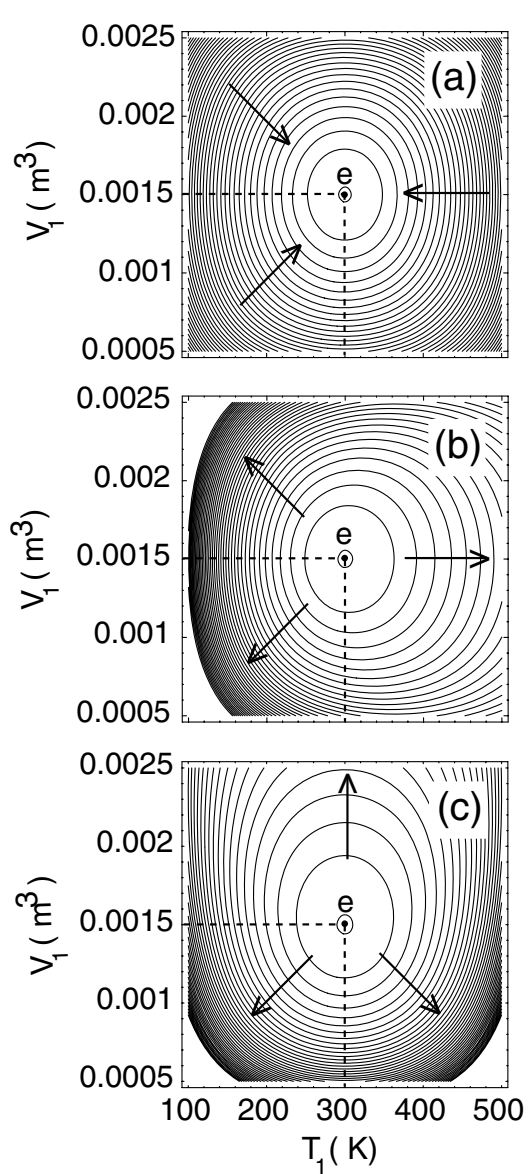

Figure 2. (a) Contour plot of the function $\left(S-S_{0}\right)_{U, V}$ (equation (11)) for the system of figure 1 with $n_{1}=n_{2}=0.1 \mathrm{~mol}$ of a monatomic gas $(c=3 / 2), A=60 \mathrm{~mol} \mathrm{~K}$ and $B=0.003 \mathrm{~m}^{3}$. (b) The same for the function $U_{S, V}$ (equation (25)) with $n_{1}=n_{2}=0.1 \mathrm{~mol}$ of a monatomic gas $(c=3 / 2)$, $C=0.003 \mathrm{~m}^{3}$ and $D=70.65 \mathrm{~K}^{3} \mathrm{~mol}^{6}$. (c) The same for the function $V_{S, U}$ (equation (30)) with $n_{1}=n_{2}=0.1 \mathrm{~mol}$ of a monatomic gas $(c=3 / 2), D=70.65 \mathrm{~K}^{3} \mathrm{~mol}^{6}$ and $E=60 \mathrm{~mol} \mathrm{~K}$. The arrows point to increasing values for the contour lines.

\subsection{Application of the extremum principles from the same initial constrained equilibrium state}

The three extremum principles considered in this work predict the same final equilibrium state, i.e. the extremum principles are equivalent. This equivalence is only meaningful for the processes $U=U_{i}=$ constant and $V=V_{i}=$ constant for the entropy maximum principle, $S=S_{e}=$ constant and $V=V_{e}=V_{i}=$ constant for the internal energy minimum principle, and $S=S_{e}=$ constant and $U=U_{e}=U_{i}=$ constant for the volume minimum principle. However, it seems interesting to remark that if one starts from the same initial constrained equilibrium state $i$ each extremum principle leads to a different final equilibrium state. For instance, in this case, the constraints for the total entropy and volume allow us to obtain

$$
U_{S, V} \equiv U\left(T_{1}, V_{1}\right)=c R\left[n_{1} T_{1}+\frac{n_{2} D_{i}^{1 / c}}{T_{1}^{n_{1} / n_{2}} V_{1}^{n_{1} / c n_{2}}\left(B-V_{1}\right)^{1 / c}}\right]
$$


instead of equation (25), for performing the minimization process. In equation (31) the constant $B$ is given by equation (10), while the constant $D_{i}$ is given by

$$
D_{i}=T_{1, i}^{c n_{1} / n_{2}} T_{2, i}^{c} V_{1, i}^{n_{1} / n_{2}} V_{2, i} \neq D
$$

One easily checks that the function defined in equation (31) presents a local minimum at volume

$$
V_{1, e^{*}}=\frac{n_{1} B}{n}=V_{1, e}
$$

and temperature

$$
T_{1, e^{*}}=\frac{D_{i}^{n_{2} / c n}}{V_{1, e^{*}}^{n_{1} / c n}\left(B-V_{1, e^{*}}\right)^{n_{2} / c n}} \neq T_{1, e} .
$$

Analogously, by fixing the total entropy and internal energy that correspond to the initial constrained equilibrium state $i$, the minimization process for the total volume is based on the function

$$
V_{S, U} \equiv V\left(T_{1}, V_{1}\right)=V_{1}+\frac{n_{2}^{c} D_{i}}{T_{1}^{c n_{1} / n_{2}}\left(A-T_{1}\right)^{c} V_{1}^{n_{1} / n_{2}}}
$$

instead of equation (30), with $A$ given by equation (9) and $D_{i}$ given by equation (32). One easily checks that the function defined in equation (35) displays a local minimum at temperature

$$
T_{1, e^{* *}}=\frac{A}{n}=T_{1, e},
$$

and volume

$$
V_{1, e^{* *}}=\frac{n_{1}^{n_{2} / n} n_{2}^{(c-1) n_{2} / n} D_{i}^{n_{2} / n}}{T_{1, e^{* *}}^{c n_{1} / n}\left(A-n_{1} T_{1, e^{* * *}}\right)^{c n_{2} / n}} \neq V_{1, e} .
$$

Figures 3(a) and (b) show contour plots for the internal energy function (31) and for the volume function (35), respectively, with a value of $D_{i}=22.36 \mathrm{~K}^{3} \mathrm{~m}^{6}$ obtained from equation (32). These plots show that the internal energy function (31) now displays an extremum (minimum) at $V_{1, e^{*}}=0.0015 \mathrm{~m}^{3}$ and $T_{1, e^{*}}=215 \mathrm{~K}$, in agreement with the values given by equations (33) and (34), respectively, while the volume function (35) presents an extremum (minimum) at $T_{1, e^{* *}}=300 \mathrm{~K}$ and $V_{1, e^{* *}}=0.00091 \mathrm{~m}^{3}$, in agreement with the values given by equations (36) and (37), respectively. We note that starting from the same initial constrained equilibrium state the internal energy extremum principle leads to a final equilibrium state with the same volume $\left(V_{1, e^{*}}=V_{1, e}\right)$ but different temperature $\left(T_{1, e^{*}} \neq T_{1, e}\right)$ than those obtained by applying the entropy extremum principle; conversely, the volume extremum principle leads to a final equilibrium state with the same temperature $\left(T_{1, e^{* *}}=T_{1, e}\right)$ but different volume $\left(V_{1, e^{* *}} \neq V_{1, e}\right)$ than those obtained by applying the entropy extremum principle. These facts become apparent by comparison of figures 2 and 3 .

\section{A phenomenological thermodynamic model}

In the previous section we have solved, from a purely thermostatic viewpoint, the problem of obtaining the final equilibrium state of the composite system of figure 1 in terms of a known initial constrained equilibrium state. In the present section we shall use a phenomenological thermodynamic approach to describe the time evolution of the temperatures and volumes of the gases toward the final equilibrium state given by equations (12) and (13). The present treatment is based on the model proposed by Gruber [12] for the problem of two ideal gases separated by an internal movable, adiabatic or diathermic, piston. The basic assumption of Gruber's model is that at any instant the state of the system can be entirely characterized by three variables $\left(T_{1}, V_{1}, \dot{V}_{1}\right)$ where $\dot{V}_{1}=\mathrm{d} V_{1} / \mathrm{d} t$. Alternatively, since $V_{1}=\mathcal{A} x$, where $\mathcal{A}$ is the 


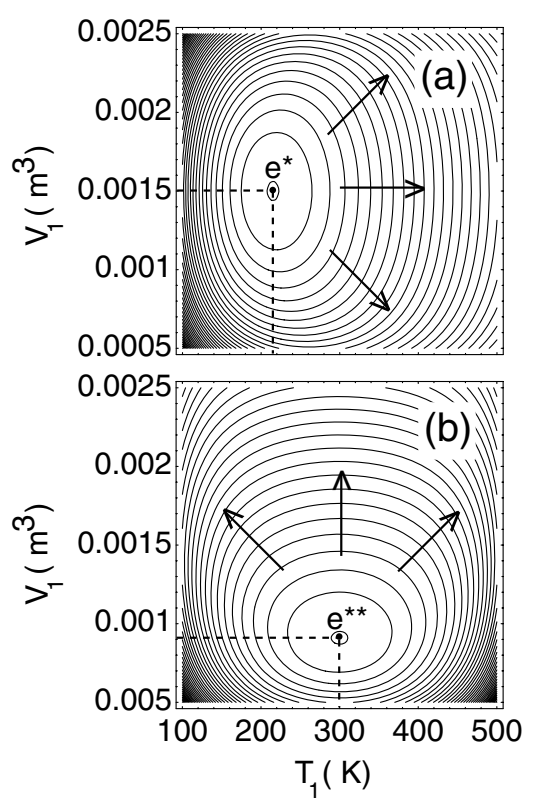

Figure 3. (a) Contour plot of the function $U_{S, V}$ (equation (31)) for the system of figure 1 with $n_{1}=n_{2}=0.1 \mathrm{~mol}$ of a monatomic gas $(c=3 / 2), B=0.003 \mathrm{~m}^{3}$ and $D_{i}=22.36 \mathrm{~K}^{3} \mathrm{~mol}^{6}$. (b) Contour plot of the function $V_{S, U}$ (equation (35)) for the system of figure 2 with $n_{1}=n_{2}=$ $0.1 \mathrm{~mol}$ of a monatomic gas $(c=3 / 2), D_{i}=22.36 \mathrm{~K}^{3} \mathrm{~mol}^{6}$ and $A=60 \mathrm{~mol} \mathrm{~K}$. The arrows point to increasing values for the contour lines.

area of the cylinder and $x$ is the macroscopic position of the piston (see figure 1), the state of the fluid can also be described by the variables $\left(T_{1}, x, v\right)$ where $v=\mathrm{d} x / \mathrm{d} t$. By using the first and second laws of thermodynamics Gruber derived the following time evolution equations for the movable diathermic piston problem $[12,13]$ :

$$
\begin{aligned}
& \frac{\mathrm{d} U_{1}}{\mathrm{~d} t}=-P_{1} \mathcal{A} v+\lambda_{1} v^{2}+\kappa\left(T_{2}-T_{1}\right), \\
& \frac{\mathrm{d} U_{2}}{\mathrm{~d} t}=P_{2} \mathcal{A} v+\lambda_{2} v^{2}-\kappa\left(T_{2}-T_{1}\right), \\
& M \frac{\mathrm{d} v}{\mathrm{~d} t}=\left(P_{1}-P_{2}\right) \mathcal{A}-\left(\lambda_{1}+\lambda_{2}\right) v,
\end{aligned}
$$

where $M$ is the mass of the piston (the mass of the gas is assumed to be negligible compared with the mass of the piston) and $\lambda_{1}, \lambda_{2}$ and $\kappa$ are phenomenological coefficients that depend on the time through $T_{1}, x$ and $v$. We note that equation (40) has the same form as the deterministic part of the Langevin equation for a Brownian particle of mass $M$ under an external force $\left(P_{1}-P_{2}\right) \mathcal{A}$. Therefore the functions $\lambda_{1}$ and $\lambda_{2}$ can be interpreted as friction coefficients arising from the collisions of the gas particles against the piston. On the other hand for $\kappa=0$ equations (38)-(40) become the time evolution equations corresponding to the adiabatic movable piston problem [12]. Therefore $\kappa$ can be interpreted as the heat conductivity associated to the heat transfer through the diathermic piston. The remaining symbols in equations (38)(40) have their usual meaning.

One easily checks that equations (38)-(40) verify energy conservation, i.e.

$$
U_{1}+U_{2}+\frac{1}{2} M v^{2}=\text { constant }
$$

where $M v^{2} / 2$ is the kinetic energy of the piston. At this point it should be noted that Gruber's model implicitly assumes that the internal energy of the diathermic piston remains constant 
along the evolution process. Taking into account equations (2) for an ideal gas and considering that the piston is at rest at time $t=0$, equation (41) reads

$$
c n_{1} R T_{1}+c n_{2} R T_{2}+\frac{1}{2} M v^{2}=c n_{1} R T_{1, i}+c n_{2} R T_{2, i}=c R A,
$$

where $T_{1, i}=T_{1}(0)$ and $T_{2, i}=T_{2}(0)$ and $A$ is given by equation (9). From equation (42) one obtains

$$
T_{2}=\frac{A-n_{1} T_{1}}{n_{2}}-\frac{M}{2 c n_{2} R} v^{2}
$$

Taking into account equations (1) and (2) for the pressure and internal energy of an ideal gas, and using equations (41) and (42), the complete set of time evolution equations is

$$
\begin{aligned}
\frac{\mathrm{d} T_{1}}{\mathrm{~d} t} & =-\frac{T_{1}}{c x} v+\frac{\lambda_{1}}{c n_{1} R} v^{2}+\frac{\kappa}{c n_{1} R}\left(T_{2}-T_{1}\right), \\
\frac{\mathrm{d} x}{\mathrm{~d} t} & =v, \\
\frac{\mathrm{d} v}{\mathrm{~d} t} & =\frac{R}{M}\left(\frac{n_{1} T_{1}}{x}-\frac{n_{2} T_{2}}{L-x}\right)-\frac{\lambda_{1}+\lambda_{2}}{M} v,
\end{aligned}
$$

with $T_{2}$ given by equation (43).

Equations (44)-(46) allow one to obtain the steady state (ss) values of $T_{1}, x$ and $v$. By setting all the time derivatives equal to zero in equations (44)-(46) one obtains

$$
v_{s s}=0, \quad T_{1, s s}=T_{2, s s}=\frac{A}{n}, \quad \frac{n_{1} T_{1, s s}}{x_{s s}}=\frac{n_{2} T_{2, s s}}{L-x_{s s}},
$$

for the values of $v, T_{1}$ and $x$ at the final equilibrium state corresponding to the piston at rest and equal temperatures and pressures at both sides. From the last equality in equation (47) one obtains $x_{s s}=n_{1} L / n$ and $V_{1, s s}=\mathcal{A} x_{s s}=n_{1} B / n$, with $B=\mathcal{A} L=V_{1, i}+V_{2, i}$ and $n=n_{1}+n_{2}$. Therefore, the model predicts the same final equilibrium state as that of equations (12) and (13) which were obtained from the extremum principles.

In order to obtain a numerical solution of equations (44)-(46) we introduce the dimensionless variables

$$
\theta_{1,2}=\frac{n T_{1,2}}{A}, \quad \xi=\frac{x}{L} \quad \tau=\frac{t}{t_{a}}, \quad v=\frac{t_{a}}{L} v,
$$

with $t_{a}=\left(M L^{2} / R A\right)^{1 / 2}$. Then, equation (43) becomes

$$
\theta_{2}=\frac{n-n_{1} \theta_{1}}{n_{2}}-\frac{n}{2 c n_{2}} v^{2}
$$

and equations (44)-(46) take the dimensionless forms

$$
\begin{aligned}
& \frac{\mathrm{d} \theta_{1}}{\mathrm{~d} \tau}=-\frac{\theta_{1}}{c \xi} v+\frac{n}{c}\left(\frac{\alpha_{1}}{n_{1}}-\frac{\delta}{2 c n_{2}}\right) v^{2}+\frac{\delta}{c}\left(1-\theta_{1}\right), \\
& \frac{\mathrm{d} \xi}{\mathrm{d} \tau}=v, \\
& \frac{\mathrm{d} v}{\mathrm{~d} \tau}=\frac{n_{1}}{n} \frac{\theta_{1}}{\xi(1-\xi)}-\frac{1}{1-\xi}\left(1-\frac{v^{2}}{2 c}\right)-\left(\alpha_{1}+\alpha_{2}\right) v,
\end{aligned}
$$

where

$$
\alpha_{1,2}=\frac{\lambda_{1,2} t_{a}}{M}, \quad \delta=\frac{\kappa t_{a}}{n_{1} R}
$$

are functions that depend on the normalized time $\tau$ through the dimensionless variables $\theta_{1}, \xi$ and $v$. By setting all the derivatives equal to zero in equations (50)-(52) one obtains 

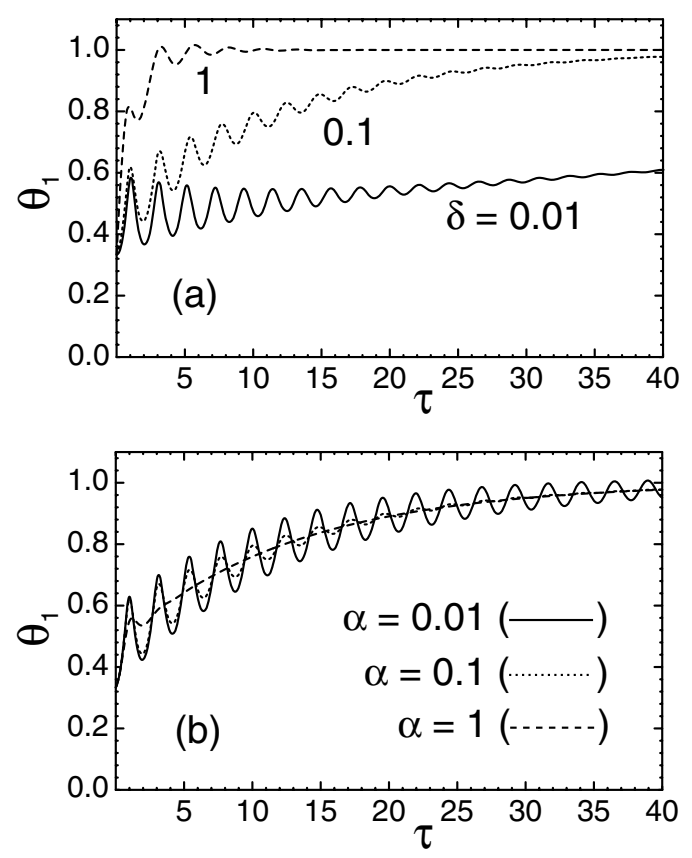

Figure 4. Plot of the normalized temperature $\theta_{1}(\tau)$ obtained from the numerical solution of equations (50)-(52) for $c=3 / 2, n_{1}=n_{2}=0.1$ and initial conditions $\xi(0)=1 / 3, \theta_{1}(0)=1 / 3$ and $v(0)=0$. (a) $\alpha=\alpha_{1}=\alpha_{2}=0.1$ and different values of the parameter $\delta(0.01,0.1$ and 1$)$; (b) $\delta=0.1$ and different values of the parameter $\alpha=\alpha_{1}=\alpha_{2}(0.01,0.1$ and 1$)$.

$$
\theta_{1, s s}=1, \quad \xi_{s s}=\frac{n_{1}}{n}, \quad v_{s s}=0
$$

for the values of $\theta_{1}, \xi$ and $v$ at the final equilibrium state.

The numerical solution of equations (50)-(52) requires knowledge of the dependence on the variables $\left(\theta_{1}, \xi, v\right)$ of the parameters $\alpha_{1}, \alpha_{2}$ and $\delta$, given by equation (53). These functions cannot be obtained from macroscopic considerations and one has to resort to microscopic models that account for the (inelastic) collisions of the gas particles against the piston. Since we are only interested in a qualitative analysis of the time evolution of the system toward the final equilibrium state, for simplicity, we assume that these parameters are constant. The time dependence of the normalized temperature $\theta_{1}$ obtained from the numerical solution of equations (50)-(52) with $\alpha=\alpha_{1}=\alpha_{2}=$ constant and $\delta=$ constant is reported in figures 4(a) and (b), for the case of a monatomic gas $(c=3 / 2)$ and $n_{1}=n_{2}=0.1 \mathrm{~mol}$. If the parameters $\delta$ and $\alpha$ are zero the system will oscillate. However, if $\delta \neq 0$ and $\alpha \neq 0$ the considered model predicts a damped oscillatory behaviour toward the final equilibrium state $\theta_{1, s s}=1$, with a decreasing relaxation time with increasing $\delta$ (see figure 4(a)), i.e. as the heat conductivity of the piston increases. We note that this relaxation time is independent of the friction parameter $\alpha$ (see figure 4(b)). However, the damping of the oscillatory behaviour becomes more pronounced as both $\delta$ and $\alpha$ increase (see figures 4(a) and (b), respectively). Therefore, the thermal equilibration of the system is characterized by a timescale of order $\tau_{t h} \sim 1 / \delta$. If one considers the system of section 3.4 with a cylinder of length $L=0.3 \mathrm{~m}$ and a steel piston (area $\mathcal{A}=0.01 \mathrm{~m}^{2}$, thickness $d=0.01 \mathrm{~m}$ and thermal conductivity $K=50 \mathrm{~W} \mathrm{~m}^{-1} \mathrm{~K}^{-1}$, so that $\kappa=K \mathcal{A} / d=50 \mathrm{~W} \mathrm{~K}^{-1}$ ), one obtains $t_{\text {th }}=\tau_{\text {th }} t_{a} \sim t_{a} / \delta=n_{1} R / \kappa=0.016 \mathrm{~s}$. This very small timescale seems to be unrealistic and this is due to the use of a very simple qualitative model that does not account for the thermal conduction in the fluid. 


\section{Summary}

In this paper we have applied three different thermodynamic extremum principles to analyse the equilibrium state in a composite system consisting of two identical ideal gases in a cylinder separated into two regions by a movable, frictionless, diathermic piston. This system has two independent internal variables. The three thermodynamic extremum principles predict final equilibrium states of equal temperatures and pressures on both sides, but these final equilibrium states only coincide when the maximization or minimization processes concerning these extremum principles are used by considering appropriate initial constrained equilibrium states. We have also outlined the main characteristics of the time evolution for the system by means of a phenomenological model that predicts the same final equilibrium state.

\section{Acknowledgments}

SV thanks the Comisión Interministerial de Ciencia y Tecnologa (CICYT) of Spain for financial support under grant no PB 98-0261 and the Junta de CyL-FSE of Spain for financial support under grant no SA097/01.

\section{References}

[1] Gibbs J W 1961 The Scientific Papers of J W Gibbs Thermodynamics vol 1 (New York: Dover) p 56, 65

[2] Tisza L 1966 Generalized Thermodynamics (Cambridge, MA: MIT Press) pp 41-8

[3] Kestin J V 1979 A Course of Thermodynamics vol 2 (New York: McGraw-Hill) ch 14 (revised printing)

[4] Callen H B 1985 Thermodynamics and an Introduction to Thermostatistics 2nd edn (New York: Wiley) ch 5, 8

[5] Bailyn M V A Survey of Thermodynamics (New York: American Institute of Physics) pp 223-32

[6] Kazes E and Cutler P H 1988 Implications of the entropy maximum principle Am. J. Phys. 56 560-1

[7] Bailyn M V A Survey of Thermodynamics (New York: American Institute of Physics) p 236

[8] Dunning-Davies J 1993 Comment on 'Implications of the entropy maximum principle' by Kazes E and Cutler P H 1988 (Am. J. Phys. 56 560-1)’ Am. J. Phys. 61 88-9

[9] Fernndez-Pineda C and Velasco S 2001 Application of thermodynamic extremum principles Am. J. Phys. 69 $1160-5$

[10] Bazarov I P 1964 Thermodynamics (Oxford: Pergamon) p 158

[11] Callen H B 1985 Thermodynamics and an Introduction to Thermostatistics 2nd edn (New York: Wiley) ch 5, 8 pp 66-8

[12] Gruber Ch 1999 Thermodynamics of systems with internal adiabatic constraints: time evolution of the adiabatic piston Eur. J. Phys. 20 259-66

[13] Gruber Ch and Frachebourg L 1999 On the adiabatic properties of a stochastic adiabatic wall: evolution, stationary non-equilibrium, and equilibrium states Physica A 272 392-428 